Published in final edited form as:

J Am Chem Soc. 2016 February 3; 138(4): 1456-1464. doi:10.1021/jacs.5b13201.

\title{
The Role of Electrostatic Interactions in Folding of $\beta$-Proteins
}

\author{
Caitlin M. Davis and R. Brian Dyer \\ Department of Chemistry, Emory University, Atlanta, Georgia 30322, United States
}

\begin{abstract}
Atomic-level molecular dynamic simulations are capable of fully folding structurally diverse proteins; however, they are limited in their ability to accurately represent electrostatic interactions. Here we have experimentally tested the role of charged residues on stability and folding kinetics of one of the most widely simulated $\beta$-proteins, the WW domain. The folding of wild type Pin 1 WW domain, which has two positively charged residues in the first turn, was compared to the fast folding mutant FiP35 Pin1, which introduces a negative charge into the first turn. A combination of FTIR spectroscopy and laser-induced temperature-jump coupled with infrared spectroscopy was used to probe changes in the amide I region. The relaxation dynamics of the peptide backbone, $\beta$-sheets and $\beta$-turns, and negatively charged aspartic acid side chain of FiP35 were measured independently by probing the corresponding bands assigned in the amide I region. Folding is initiated in the turns and the $\beta$-sheets form last. While the global folding mechanism is in good agreement with simulation predictions, we observe changes in the protonation state of aspartic acid during folding that have not been captured by simulation methods. The protonation state of aspartic acid is coupled to protein folding; the apparent $\mathrm{p} K_{\mathrm{a}}$ of aspartic acid in the folded protein is 6.4. The dynamics of the aspartic acid follow the dynamics of the intermediate phase, supporting assignment of this phase to formation of the first hairpin. These results demonstrate the importance of electrostatic interactions in turn stability and formation of extended $\beta$-sheet structures.
\end{abstract}

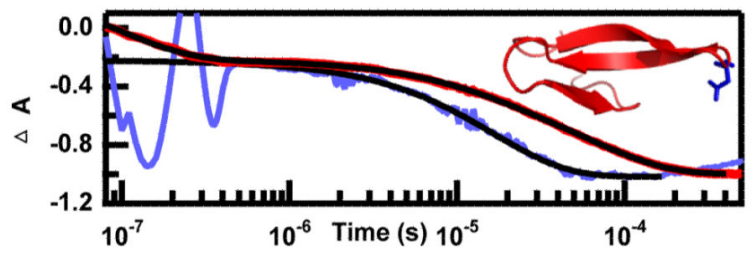

*Corresponding Author, briandyer@emory.edu. ASSOCIATED CONTENT

Supporting Information

The Supporting Information is available free of charge on the ACS Publications website at DOI: 10.1021/jacs.5b13201. Temperature dependent FTIR and difference spectra of $\mathrm{pH} 7$ Pin1 and pH 5.8 and pH 7 FiP35 WW Domains, SVD analysis of pH 7 FiP35 FTIR data, $\mathrm{pH}$ dependent fluorescence and $\mathrm{CD}$ data of FiP35, representative IR T-jump relaxation kinetics of FiP35 at low temperature, second derivative of FBP28 at $5^{\circ} \mathrm{C}$, and a complete table of relaxation kinetics for $\mathrm{pH} 7$ and $\mathrm{pH} 5.8$ at $1613,1636,1680$, and $1710 \mathrm{~cm}^{-1}$. (PDF)

Notes

The authors declare no competing financial interest. 


\section{INTRODUCTION}

A close interplay between experiment and theory in the study of protein dynamics has helped to understand better how proteins fold and function. There is still room for improvement, particularly with respect to approaches for measuring and modeling electrostatic interactions. The conventional approach in molecular dynamics simulations has been to set a fixed protonation state for each titratable amino acid residue; however, fixed $\mathrm{p} K_{\mathrm{a}}$ values likely do not appropriately describe the relationship between protonation state and conformation. Ionizable amino acid residues play a prominent role in enzyme catalysis, substrate binding, and protein folding. ${ }^{1-5}$ The $\mathrm{p} K_{\mathrm{a}}$ value of these amino acid residues is strongly dependent on the local environment, and can be shifted far from the solution value. ${ }^{6,7}$ Similarly, the same residue may have a different $\mathrm{p} K_{\mathrm{a}}$ in different protein conformations. Structurally specific experimental characterization of the ionizable residue's protonation state and protein dynamics is necessary to test and further refine computational methods. Infrared spectroscopy offers a method to not only resolve protein backbone structure and dynamics, but also provides information about the structure and environment of the amino acid side chains. ${ }^{8}$ The amide I mode of the peptide backbone is sensitive to secondary structure; characteristic infrared bands can be assigned to solvated and buried $a$ helix, $\beta$ sheets, turns and random coil structures. The carbonyl group of protonated carboxyl groups can be unambiguously assigned, because it occurs in a region free from overlapping absorption by other groups. This provides a mechanism for monitoring protonation and deprotonation of carboxyl groups of interest. We have previously employed time-resolved infrared spectroscopy to probe the time-dependent protonation state of a carboxylic acid side chain in a large protein with single-residue specificity without incorporating any labels. ${ }^{9-11}$ Thus, it should be possible to determine the coupling between protonation state and protein conformation using time-resolved infrared spectroscopy of the peptide backbone and carboxylic acid side chains.

Here we have experimentally tested the role of charged residues on stability and folding kinetics of one of the most widely simulated $\beta$-protein families, the WW domain. The WW domain family consists of an antiparallel and highly twisted three-stranded $\beta$-sheet structure with a small hydrophobic core and two highly conserved tryptophan residues. ${ }^{12-15}$ Because of their fast folding rates and simple structure, WW domains have been the focus of extensive computational and experimental studies. ${ }^{16-29}$ Many of these studies have predicted a folding mechanism where folding is initiated in the turn of the first hairpin. ${ }^{17,18,23,25,27,28,30,31}$ A comparison of the sequence and stability of these WW domains reveals the presence of several charged residues in the first turn (Table 1). In the native sequences, the first turn is flanked on each side by amino acids with positively charged side chains (blue). WW domains that also have an amino acid with a negatively charged side chain (red) in the first turn have a melting temperature of $\sim 10{ }^{\circ} \mathrm{C}$ higher than related sequences. ${ }^{34}$ One possibility is that these charged side chains form a salt bridge that stabilizes the first turn. This interaction depends on the protonation state of the negatively charged side chain.

We have addressed this issue by studying the role of aspartic acid and arginine on stability and folding kinetics in the Pin1 family of WW domains (Figure 1). We investigated three 
WW domains: one without a negatively charged residue in the turn (Pin1), one with both negative and positively charged residues in the turn (FiP35), and one without the positively charged flanking residues in the turn (R12A R16A $\Delta \Delta$ FiP35). We found that while the $\Delta \Delta$ FiP35 mutant forms a WW Domain, mutation of Arg 12 and Arg 16 resulted in destabilization of the peptide and an increase in propensity to aggregate. Equilibrium infrared spectroscopy revealed that aspartic acid was protonated under folded conditions in both FiP35 and $\Delta \Delta$ FiP35 WW domains, making it unlikely that arginine and aspartic acid form a salt bridge. The $\mathrm{pH}$ titration of Asp13 is broad, likely because its $\mathrm{p} K_{\mathrm{a}}$ is coupled with the protein conformation. The dynamics of the FiP35 backbone and Asp13 side chain were measured using temperature jump, time-resolved infrared spectroscopy. Pulsed laser excitation was used to rapidly initiate a shift in the folding equilibrium. The relaxation of the WW domain was measured by independently probing the components of the IR amide I band assigned to the $\beta$-sheets and $\beta$-turn and the protonated carboxyl side chain of aspartic acid.

FiP35 exhibited multiexponential behavior when the carbonyl backbone was probed, but only one phase when the aspartic acid was probed. Similar to other WW domains, wavelength dependent infrared measurements in the amide I region reveal a fast $100 \mathrm{~ns}$ phase, intermediate $\sim 10 \mu$ s phase and a slower $\sim 100 \mu$ s phase. ${ }^{27,28}$ The kinetics of the aspartic acid protonation state follow the kinetics of the intermediate phase when the backbone is probed, supporting the assignment of this phase to formation of the first hairpin. While the global folding mechanism agrees with computer simulations that predict folding that initiates in the first turn, these simulations methods were incapable of modeling protonation changes necessary to describe the local environment of the aspartic acid in the turn. These results demonstrate the importance of developing experimental and computational methods that characterize electrostatic interactions in proteins.

\section{EXPERIMENTAL SECTION}

\section{Protein Synthesis and Purification}

FiP35, $\Delta \Delta$ FiP35, and Pin1 were synthesized via standard 9-fluorenylmethyloxycarbonyl (Fmoc) base solid-phase chemistry on a Liberty1 microwave peptide-synthesizer (CEM, Matthews, NC). Fmoc-PAL-PS resin (Applied Biosystems, Foster City, CA) was used to form a peptide amide. The peptide was purified by reverse-phase chromatography (C18 column) using a water/acetonitrile gradient with $0.1 \%$ trifluoroacetic acid (TFA) as the counterion. TFA interferes in the Amide-I IR measurements at $1672 \mathrm{~cm}^{-1}$. The peptide was dissolved in a $2 \mathrm{mM} \mathrm{HCl}$ solution and lyophilized to allow exchange of the TFA counterion for $\mathrm{HCl}^{35}$ The identity of the peptide was confirmed by matrix-assisted laser desorption ionization time-of-flight mass spectrometry. The peptide was lyophilized and dissolved in $\mathrm{D}_{2} \mathrm{O}$ to allow deuterium-hydrogen exchange of amide protons. The peptide was lyophilized a second time and resuspended in $\mathrm{D}_{2} \mathrm{O}$ buffer. Buffers were prepared at $20 \mathrm{mM}$ concentration for the appropriate $\mathrm{pH}$ range: acetate $(\mathrm{pH} 4-5.5)$, potassium phosphate $(\mathrm{pH} 6-$ 8), Tris- $\mathrm{HCl}(\mathrm{pH} 8.5-9)$, and carbonate-bicarbonate (9.5-10.5). Sample concentrations of 0.05-2.0 $\mathrm{mM}$ were prepared for $\mathrm{CD}$, fluorescence and IR experiments. 


\section{Spectroscopy}

CD wavelength scans and CD melting curves were recorded on a Jasco J-810

spectropolarimeter equipped with a PFD-425S Jasco temperature controller module (Jasco, Inc., Easton, MD). Peptides were dissolved at $50 \mu \mathrm{M}$ in buffer. All measurements were obtained using a $1 \mathrm{~mm}$ path length cell. Wavelength scans were recorded over the range of 260 to $190 \mathrm{~nm}$ with an average of 3 repeats. During spectral acquisition, a bandwidth of 2 $\mathrm{nm}$ and scan rate of $50 \mathrm{~nm} / \mathrm{min}$ was used. Thermal unfolding experiments were performed by monitoring the signal at $226 \mathrm{~nm}$ from 5 to $95^{\circ} \mathrm{C}$ using a $0.1^{\circ} \mathrm{C}$ interval and scan rate of $30{ }^{\circ} \mathrm{C} / \mathrm{h}$. During the thermal unfolding experiment a full wavelength scan was obtained every $5{ }^{\circ} \mathrm{C}$ after a $60 \mathrm{~s}$ delay. The buffer and protein concentrations were the same as used in the wavelength scan experiments.

\section{FTIR Spectroscopy}

The equilibrium melting behavior was monitored on a Varian Excalibur 3100 FTIR spectrometer (Varian Inc., Palo Alto, USA) using a temperature controlled IR cell. The IR cell consists of two $\mathrm{CaF}_{2}$ windows stacked and separated by a $100 \mu \mathrm{m}$ Teflon spacer split into two compartments, a sample and a reference. The same cells are used for equilibrium FTIR and T-jump experiments. No aggregation was observed in the infrared at reported concentrations. All spectra shown at a specific temperature are obtained from the ratio of the single beam spectra of sample solution with protein to the reference buffer solution without protein. The temperature-dependent difference spectra were then generated by subtracting the spectrum at the lowest temperature from the spectra at higher temperatures. Data collected at $50 \mu \mathrm{M}$ concentration were baseline corrected using a baseline spline fit. The second derivative spectra were computed in IGOR PRO after smoothing the data with a sixth order binomial algorithm to remove any residual water vapor (WaveMetrics, Lake Oswego, OR).

\section{Time Resolved Temperature Jump (T-Jump) Relaxation Measurements}

The IR T-jump apparatus has been described previously. ${ }^{36}$ Pulsed laser excitation is used to rapidly perturb the folding equilibrium on a time scale faster than the molecular dynamics of interest. Time resolved infrared is then used to probe the reaction. A Q-switched GCR-4 Nd:YAG laser (Spectra Physics, Mountainview, CA) fundamental at $1064 \mathrm{~nm}$ is Raman shifted (one Stokes shift in $200 \mathrm{psi}_{2}$ gas) to produce a $10 \mathrm{~ns}$ pulse at $2 \mu \mathrm{m}$. The magnitude of the T-jump is calculated using the change in reference absorbance with temperature. IR T-jump experiments were conducted at multiple concentrations between 0.5 and $2.0 \mathrm{mM}$. The T-jump reference is taken from $\mathrm{D}_{2} \mathrm{O}$ buffer with $20 \mathrm{mM}$ potassium phosphate buffer at pD* 7.0 at the same temperature and frequency as the sample. Absorbance changes at the reference frequency are due only to changes in $\mathrm{D}_{2} \mathrm{O}$ absorbance, which is used as an internal thermometer. ${ }^{36}$

The change in signal induced by the T-jump is probed in real time by a continuous laser with a frequency in the amide I' band of the IR. The mid-IR probe beam is generated by a continuous wave quantum cascade laser (Daylight Solutions Inc., San Diego, CA) with a tunable output range of $1570-1730 \mathrm{~cm}^{-1}$. The transient transmission of the probe beam through the sample is measured using a fast, $100 \mathrm{MHz}$, photovoltaic MCT IR detector/ 
preamplifier (Kolmar Technologies, Newburyport, MA). Transient signals are digitized and signal averaged (1000 shots) using a Tektronics digitizer (7612D, Beaverton, OR).

Instrument control and data collection are controlled using a LabVIEW computer program.

\section{Analysis of Kinetics Data}

The peptide relaxation kinetics must be deconvolved from the observed kinetics. Accurate deconvolution is possible as the instrument response is determined from the reference measurement under the exact conditions of the sample measurements. In order to minimize detector artifacts, the reference is scaled prior to subtraction from the sample. The decay function is a multiexponential decay with the formula

$$
A=A_{0}+\ldots+A_{n} \exp \left(\frac{-\left(x-x_{0}\right)}{\tau_{n}}\right)
$$

where $A_{0}$ is an offset, $n$ is the number of exponentials to fit, $A_{n}$ is a preexponential factor, $\tau_{n}$ is the relaxation lifetime of the sample and $x_{0}$ is the time offset. In order to best fit the data, the minimum number of exponentials with unique relaxation lifetimes was selected. The data are fit over the interval from $80 \mathrm{~ns}$ to an order of magnitude outside the slowest exponential. The data analysis was performed in IGOR PRO (WaveMetrics, Lake Oswego, $\mathrm{OR})$.

\section{RESULTS AND DISCUSSION}

\section{Far-UV CD Spectroscopy}

The Pin1, FiP35 and $\Delta \Delta$ FiP35 WW domains were synthesized to study the aspartic acid in loop 1 of FiP35 and its interaction with neighboring arginine residues. Far-UV CD is a good indicator of peptide secondary structure. Typically, $\beta$-sheet peptides have a CD spectrum with a negative peak at $\sim 218 \mathrm{~nm}$ and a positive peak at $\sim 195 \mathrm{~nm} .{ }^{37}$ Folded WW domains instead have a CD spectrum dominated by a negative peak at $\sim 202 \mathrm{~nm}$ and a positive peak at $\sim 230 \mathrm{~nm} .{ }^{38,39}$ Small variations in the position and intensities of these peaks have been observed among the WW domain family. ${ }^{38}$ The peak at $\sim 202 \mathrm{~nm}$ resembles the random coil peak usually found at $200 \mathrm{~nm}$. Disorder in the $\mathrm{N}$ - and C-termini of the folded structure of WW domains is thought to contribute to the negative peak in the CD spectrum. ${ }^{39-42}$ The peak at $\sim 230 \mathrm{~nm}$ arises from the presence of ordered aromatic side chains. ${ }^{18,37,39}$ Folded Pin1 WW domains and its mutants have been shown to have additional structure between 190 and $210 \mathrm{~nm} .^{33,43}$ The far-UV CD spectra of the Pin1, FiP35 and $\Delta \Delta$ FiP35 WW domains are consistent with the Pin1 family WW domain secondary structure (Figure 2A).

Thermal denaturation was monitored by recording the ellipticity change at $226 \mathrm{~nm}$ with temperature (Figure 2B). The Pin1 and FiP35 WW domains exhibit the typical heat induced unfolding behavior with a loss of intensity at $226 \mathrm{~nm}$ and a shift of the minimum at 197 to $200 \mathrm{~nm}$, corresponding to a change in the secondary structure from WW domain to random coil (Figure 2A) ${ }^{39}$ In addition to the peaks associated with thermal unfolding, the $\Delta \Delta$ FiP35 mutant gains a negative peak at $\sim 219 \mathrm{~nm}$ and positive peak at $\sim 190 \mathrm{~nm}$, consistent with $\beta$ sheet structure. This is evidence for formation of $\beta$-sheet aggregates at high temperature. 
The melting curves of FiP35 and Pin1 (Figure 2B) were fit to an apparent two-state equilibrium model:

$$
A_{0}=\frac{A_{\mathrm{f}}+S_{\mathrm{f}} T}{1+\exp \left(-\frac{\Delta H}{R}\left(\frac{1}{T}-\frac{1}{T_{\mathrm{m}}}\right)\right)}+\frac{\left(A_{\mathrm{u}}+S_{\mathrm{u}} T\right)\left(\exp \left(-\frac{\Delta H}{R}\left(\frac{1}{T}-\frac{1}{T_{\mathrm{m}}}\right)\right)\right)}{1+\exp \left(-\frac{\Delta H}{R}\left(\frac{1}{T}-\frac{1}{T_{\mathrm{m}}}\right)\right)}
$$

where $A_{0}$ is the observed absorbance, $A_{\mathrm{f}}$ and $A_{\mathrm{u}}$ are the absorbance contributions from the folded and unfolded populations at $0{ }^{\circ} \mathrm{C}, S_{\mathrm{f}}$ and $S_{\mathrm{u}}$ are the slope of the folded and unfolded state baseline, $\Delta H$ is the enthalpy change at the midpoint, $\mathrm{R}$ is the gas constant, and $T_{\mathrm{m}}$ is the transition midpoint. ${ }^{44}$ This analysis assumes a $\Delta C_{\mathrm{p}}$ of 0 as it is unlikely that a small peptide like a WW domain would have a large difference in heat capacity between the folded and unfolded states. The data are then normalized for comparison. The observed melting temperature of Pin 1 is $57.1 \pm 0.1^{\circ} \mathrm{C}$. This agrees with the previously reported melting temperature of Pin $1,58.6{ }^{\circ} \mathrm{C}$, obtained by a CD melt monitored at $226 \mathrm{~nm} .{ }^{43}$ The melt of FiP35 is less cooperative, with a melting temperature of $80^{\circ} \mathrm{C} \pm 1{ }^{\circ} \mathrm{C}$. Because of the high transition temperature and the breadth of the transition it was not possible to observe a post transition baseline. However, the observed melting temperature agrees with the previously reported melting temperature of FiP35, $78^{\circ} \mathrm{C}$, which was obtained by global fit of thermaldenaturant $\mathrm{CD}$ melting curves monitored at $227 \mathrm{~nm} \cdot{ }^{16,26}$

\section{FTIR Spectroscopy}

The temperature-induced unfolding of Pin1 and FiP35 was studied over the range from 10 to $90{ }^{\circ} \mathrm{C}$ in $5{ }^{\circ} \mathrm{C}$ intervals using FTIR spectroscopy monitored in the amide I' region. To avoid the aggregation of $\Delta \Delta \mathrm{FiP} 35$ observed at high temperature by $\mathrm{CD}$, the FTIR absorption spectrum was collected at $50 \mu \mathrm{M}$ at $20^{\circ} \mathrm{C}$. The high and low temperature absorption spectra of the amide I' spectral region (amide I region of peptides in $\mathrm{D}_{2} \mathrm{O}$ ) of the peptides are shown in Figure 3A (complete temperature dependent spectra are shown in Supporting Information Figure $\mathrm{S} 1$ ). The amide I' absorbance arises from the $\mathrm{C}=\mathrm{O}$ stretching vibration of the polypeptide backbone carbonyls, and is an established indicator of secondary structure. ${ }^{45-47}$ This relatively broad band contains contributions from the entire polypeptide backbone, which in the case of WW domains includes $\beta$-sheet, $\beta$-turn and random coil structure. The changes with temperature are highlighted by the difference spectra for each peptide (Figure 3B). The difference spectra are generated by subtracting the lowest temperature spectrum from each absorption spectrum at higher temperature. Negative peaks correspond to specific structures or interactions present in the folded state, and positive peaks correspond to new interactions with solvent in the unfolded state. The individual peaks are more easily distinguished in the second derivative of the FTIR spectra at the lowest temperature (Figure $3 \mathrm{C})$.

At low temperature there are three main components of the amide I' band, centered at 1613, 1636 , and $1680 \mathrm{~cm}^{-1}$. The intensity of these features decreases with increasing temperature, meaning that they are all associated with the folded state. These peaks have previously been observed in other WW domains. ${ }^{27,28,48}$ A peak at $\sim 1611 \mathrm{~cm}^{-1}$ in $\beta$-hairpins has been assigned to an amide $\mathrm{C}=\mathrm{O}$ group in the turn usually involved in multiple hydrogen bonds with side chain or backbone donors. ${ }^{49,50}$ There are three such groups in the first turn and 
one such group in the second turn of wildtype Pin1 WW domain. ${ }^{51}$ Because of this, it is not possible to independently assign the $1613 \mathrm{~cm}^{-1}$ peak to the turn of the first or second hairpin. Upon mutation of the first turn to SA-DGR, a FiP35 like mutant, one of the interactions in the first turn and the interaction in the second turn are conserved, and two new hydrogen bonds between backbone carbonyls and side chain or backbone donors arise. ${ }^{52}$ The peak is of equal intensity in each of the peptides (Figure 3C), because the number of hydrogen bonds is conserved. IR bands at 1634 and $1681 \mathrm{~cm}^{-1}$ are well established components of antiparallel $\beta$-sheets. ${ }^{53}$ The peak at $1634 \mathrm{~cm}^{-1}$ arises from inphase coupling of carbonyl groups in the sheet and the peak at $1681 \mathrm{~cm}^{-1}$ arises from outof-phase coupling of carbonyls in the sheet. The Pin1, FiP35 and $\Delta \Delta$ FiP35 amide I' bands at 1636 and $1680 \mathrm{~cm}^{-1}$ are consistent with these characteristic $\beta$-sheet markers.

Analysis of the spectral region above $1700 \mathrm{~cm}^{-1}$ reveals a peak at $1713 \mathrm{~cm}^{-1}$ that is present in the FiP35 and $\Delta \Delta$ FiP35 WW domain, but not the Pin1 WW domain. This peak arises from the $\mathrm{C}=\mathrm{O}$ stretch of a protonated carboxyl side chain, either glutamic acid or aspartic acid. ${ }^{8}$ The difference between the residues with carboxyl group side chains in the three sequences is in the first turn; FiP35 and $\Delta \Delta \mathrm{FiP} 35$ have an aspartic acid that is not found in Pin1. Therefore, the peak at $1713 \mathrm{~cm}^{-1}$ can be assigned to Asp13, most likely in its protonated form. An alternative possibility is that Asp13 is deprotonated but involved in a strong salt bridge. A deprotonated carboxylate group has two strong bands at 1400 and 1570 $\mathrm{cm}^{-1}\left(v_{\mathrm{sym}}\right.$ and $\left.v_{\text {asym }}\right)$. These positions may shift upon cation chelation, which changes bond lengths and bond angles. In unidentate chelation of the deprotonated carboxylate group, the two $\mathrm{CO}$ bonds are no longer equivalent, with the chelating oxygen having more single bond character and the nonchelating oxygen having more double bond character. In extreme cases this has been found to shift one of the bands to $1713 \mathrm{~cm}^{-1}$, similar to the band assigned to the $\mathrm{C}=\mathrm{O}$ stretch of a protonated carboxyl side chain. ${ }^{54}$ There are two arginines in FiP35 that may provide a cation through a salt bridge with aspartic acid, $\operatorname{Arg} 12$ and $\operatorname{Arg} 15$. However, mutation of these arginines to alanine in $\Delta \Delta \mathrm{FiP} 35$, does not eliminate the peak at $1713 \mathrm{~cm}^{-1}$ (Figure 3), supporting our assignment of this band to the protonated carboxylic acid side chain of Asp13. Similar to the bands in the amide I' region, the position of the $\mathrm{C}=\mathrm{O}$ stretch of the protonated aspartic acid is sensitive to hydrogen bonding. The vibrational stretching band associated with the $\mathrm{C}=\mathrm{O}$ can be observed anywhere in the region from 1713 to $1775 \mathrm{~cm}^{-1}$, with lower frequencies indicating the carbonyl side chain is engaged in hydrogen bonding. ${ }^{8}$ In the X-ray crystal structure of a R12A F28W FiP35 mutant a native contact is formed between Ser11 and Asp13. ${ }^{52}$ On this basis we can assign the $1713 \mathrm{~cm}^{-1}$ band to hydrogen bonding between the carbonyl of the protonated aspartic acid side chain and the hydroxyl of the serine.

The $\mathrm{pH}$ dependence of the protonated carboxylic acid side chain was studied over the range from $\mathrm{pH} 4-10$ (Figure 4). The $\mathrm{pH}$ was not dropped below $\mathrm{pH} 4$ to minimize contributions to the $1713 \mathrm{~cm}^{-1}$ band from the protonated glutamic acid side chain ( $\mathrm{p} K_{\mathrm{a}}$ of 4.2 ), which overlaps with the protonated aspartic acid side chain band. Measurements were carried out at $20^{\circ} \mathrm{C}$, a temperature where at neutral $\mathrm{pH}$ the peptide was found to be folded (Figure 3C). The three bands assigned to the folded WW domain, 1613, 1636, and $1680 \mathrm{~cm}^{-1}$, are observed at each $\mathrm{pH}$. Unfolding of the $\beta$-sheet is characterized by a blue shift and 
broadening of the amide I band. ${ }^{8}$ The overlap of the $1636 \mathrm{~cm}^{-1}$ band across all $\mathrm{pHs}$ demonstrates that the $\beta$-sheets are intact over the entire $\mathrm{pH}$ range (Figure 4A). However, the decrease in the relative intensity of the $1613 \mathrm{~cm}^{-1}$ band, assigned to the turn, and changes in the region between 1650 and $1680 \mathrm{~cm}^{-1}$, assigned to solvated carbonyls, indicate that there are structural changes associated with $\mathrm{pH}$ that do not affect the hydrogen bond network of the $\beta$-sheets. $\mathrm{pH}$ dependent fluorescence and circular dichroism spectra (Supporting Information Figure S4-S5) confirm that the WW domain undergoes structural rearrangement, repacking of the hydrophobic core, at high and low $\mathrm{pH}$ but that the WW domain does not completely denature. The aspartic acid is protonated to some extent over the entire $\mathrm{pH}$ range. An increase in the intensity of the turn structure, $1613 \mathrm{~cm}^{-1}$, correlates with the increase in the population of protonated aspartic acid, $1713 \mathrm{~cm}^{-1}$, as the $\mathrm{pH}$ is lowered. This is evidence that changes in the protonation state of aspartic acid, found in the first turn, affect the hydrogen-bonding network of the first turn. A pH titration of the protonated carboxylic acid side chain of Asp 13 derived from the second derivative of the $\mathrm{pH}$ dependent IR absorbance at $1713 \mathrm{~cm}^{-1}$ is shown in Figure 4B. The $\mathrm{p} K_{\mathrm{a}}$ derived from this fit is $6.4 \pm 0.9$. The $\mathrm{p} K_{\mathrm{a}}$ of the aspartic acid side chain of free aspartic acid is 3.9; however, electrostatic and hydrophobic induced $\mathrm{p} K_{\mathrm{a}}$ shifts of as many as $6 \mathrm{p} K_{\mathrm{a}}$ units have been reported for aspartic acid in proteins. ${ }^{55-58}$ The transition is broad, likely because protonation is coupled with folding of the WW domain. At neutral pH the aspartic acid completely deprotonates when the WW domain unfolds (Figure $3 \mathrm{~A}$ ). The $\mathrm{p} K_{\mathrm{a}}$ of the Asp13 side chain is dependent on both $\mathrm{pH}$ and the FiP35 WW domain secondary structure.

The normalized melting curves for Pin1 and FiP35 WW domains derived from the temperature dependent IR absorbance at 1636 and $1713 \mathrm{~cm}^{-1}$ are shown in Figure 5. The data were normalized after being fit to an apparent two-state equilibrium model using eq 2 . The melting temperature of the wildtype Pin 1 is $53.1 \pm 0.2{ }^{\circ} \mathrm{C}$. The melting temperature of FiP35 is wavelength dependent, with a relatively broad transition when probed at $1636 \mathrm{~cm}^{-1}$ and a sharper transition when probed at $1713 \mathrm{~cm}^{-1}$. When the $1636 \mathrm{~cm}^{-1}$ data is fit to the sum of two melts it fits to a $T_{\mathrm{m}}$ of $40.4 \pm 0.1{ }^{\circ} \mathrm{C}$ and $77.1 \pm 0.1{ }^{\circ} \mathrm{C}$. This agrees well with the melting temperature probed by $\mathrm{CD}$. The transition at $1713 \mathrm{~cm}^{-1}$ fits to a $T_{\mathrm{m}}$ of $59.1 \pm 0.2$ ${ }^{\circ} \mathrm{C}$. The observation of frequency dependent melt profiles suggest the presence of more than one temperature-dependent process. A combined SVD analysis and global fit (Supporting Information Figure S2) reveals that the lower temperature transition arises from melting of the sheets prior to the main transition at higher temperature.

\section{Temperature-Jump Relaxation Kinetics}

The relaxation kinetics of the folding/unfolding transition of FiP35 WW domain following a laser-induced temperature jump were probed using time-resolved infrared spectroscopy. Structure specific measurements were made using the amide I' frequency for the amide carbonyls of the turns $\left(1613 \mathrm{~cm}^{-1}\right)$, residues involved in interstrand coupling of the sheets (1636 and $1680 \mathrm{~cm}^{-1}$ ), and the protonated carboxylic acid side chain of aspartic acid (1713 $\mathrm{cm}^{-1}$ ). Jumps were performed slightly off peak center to maximize the transient absorbance signal. The complete relaxation kinetics for each frequency is reported in the Supporting Information (Table S1-S4). Time resolved measurements examined the dependence of the relaxation rates on the final temperature following a temperature jump. The magnitude of 
the temperature jump was kept constant while varying the final temperature for a range of final temperatures below the global melting transition. The observed rates reflect the sum of the forward and reverse rate constants for the reversible folding reaction. Under conditions where the protein is predominantly folded the relaxation rate is dominated by the rate of the folding reaction. Because the final temperature of all jumps is at least $20^{\circ} \mathrm{C}$ below the melting temperature, the folding rate will dominate the observed relaxation kinetics. The relaxation kinetics of the amide I' region $\left(1613,1636\right.$, and $\left.1680 \mathrm{~cm}^{-1}\right)$ are best fit by a triple exponential (eq 1). At high temperatures the $1680 \mathrm{~cm}^{-1}$ data is fit to a double exponential due to artifacts in the data at early time and the overall low absorbance change. Examples of jumps to lower temperature where there are fewer early time scale artifacts are included in the Supporting Information Figure S6. The relaxation kinetics of the aspartic acid side chain is best fit by a single exponential at all temperatures. Although aggregation was observed over the time scale of equilibrium FTIR measurements at $\mathrm{pH} 7$ (Figure S1, S2), it does not contribute to the T-jump dynamics because aggregation is slower than the duration of the Tjump, which relaxes back to the initial temperature in a few milliseconds. IR T-jump measurements at $\mathrm{pH} 5.8$, where aggregation in FTIR measurements is not observed, agree with measurements obtained at $\mathrm{pH} 7$ (Table S1-S4). Figure 6 displays the relaxation kinetics of FiP35 following a jump from 35 to $50{ }^{\circ} \mathrm{C}$. The fits of the data in Figure 6 are reported in Table 2. There is good agreement between the dynamics at each of the amide I' frequencies. There is a fast $\sim 100 \mathrm{~ns}$ phase $\left(\tau_{1}\right), \mathrm{a} \sim 10 \mu$ s phase $\left(\tau_{2}\right)$ and a slower $\sim 100 \mu$ s phase $\left(\tau_{3}\right)$. The single phase observed when the frequency assigned to the aspartic acid side chain is probed agrees with the intermediate $\tau_{2}$ phase.

Frequency dependent measurements of the amide I' region reveal differences in the dynamics of the turns $\left(1613 \mathrm{~cm}^{-1}\right)$ and $\beta$-sheets $\left(1636\right.$ and $\left.1680 \mathrm{~cm}^{-1}\right)$. Because of overlap of peaks in the amide I' region, probes at each location are sensitive to dynamics associated with folding at multiple locations, resulting in multiexponential kinetics regardless of probe frequency. It is also likely that the spectral responses are coupled such that formation of one structure affects the others. However, the relative amplitude of each kinetics phase depends on the probe frequency, which allows us to assign the structural feature that contributes most strongly to each phase. The relative amplitude of the fast phase is greatest in the transient measured at $1613 \mathrm{~cm}^{-1}$, which probes the turn of both hairpins of the WW domain. The slow phase dominates the kinetics measured at $1636 \mathrm{~cm}^{-1}$, which probes interstrand coupling across the $\beta$-sheets of the WW domain. This assignment is supported by the kinetics measured at $1680 \mathrm{~cm}^{-1}$, also dominated by the slow phase, which probes out-ofphase coupling of the carbonyl bonds in the $\beta$-sheet. Based on these observations we assign the fast phase to turn formation and the slowest phase to sheet formation.

Unlike the measurements in the amide I' region, the probe at $1713 \mathrm{~cm}^{-1}$ is sensitive to dynamics of a single side chain, aspartic acid. The peak is in a clear spectral region, easing interpretation of observed dynamics. The observed dynamics report on the folding of the first hairpin of FiP35, because the aspartic acid, located in the first turn, is only protonated when FiP35 is folded. It is likely that many of the contacts in the turn and $\beta$-sheet of the first hairpin must be formed in order for the environment to be suitable for the aspartic acid to protonate. There are many examples of elevated $\mathrm{p} K_{\mathrm{a}}$ 's of aspartic acid groups in the 
hydrophobic interior of proteins, so it is likely that protonation occurs concurrently with packing of the hydrophobic region of the first hairpin. The single exponential kinetics match the kinetics of the intermediate phase probed in the amide I' region (Table 2). An Arrhenius plot of the observed kinetics at 1713 and $1636 \mathrm{~cm}^{-1}$ demonstrates this agreement across all of the temperatures probed (Figure 7). On the basis of this close correspondence, we can assign the intermediate phase to formation of $\beta$-sheet of the first hairpin. Using observations from the frequency dependent measurements in the amide I' region and of the aspartic acid side chain it is possible to interpret the three phases observed in the Arrhenius analysis. The fast relatively temperature independent $100 \mathrm{~ns}$ phase is assigned to formation of the turns. The intermediate $\sim 10 \mu$ s phase corresponds to formation of the hydrogen bonding network in the $\beta$-sheet of the first hairpin and the slow highly temperature dependent $\sim 100 \mu$ s phase can be attributed to formation of the hydrogen bonding network in the $\beta$-sheet of the second hairpin.

Previous temperature jump studies of FiP35 probed by fluorescence spectroscopy reported biphasic behavior below $T_{\mathrm{m}}$ and single exponential behavior above $T_{\mathrm{m}}$. A jump to a final temperature of $64{ }^{\circ} \mathrm{C}$, below $T_{\mathrm{m}}$, fit to relaxation lifetimes of 1.5 and $15 \mu \mathrm{s} .{ }^{26}$ Infrared Tjumps to $60^{\circ} \mathrm{C}$ have the same intermediate and slow phases (Table S1-S4). The difference in folding behavior above and below $T_{\mathrm{m}}$ was interpreted as the onset of downhill folding. Recently, pressure jump experiments also showed FiP35 to be biphasic, but with different kinetic characteristics in the fast phase than observed by temperature jump. ${ }^{29}$ Experimental results combined with computer modeling predict folding proceeds through two parallel pathways, with the competing pathways having hairpin 1 or hairpin 2 formed first. The folding pathway perturbed by temperature and pressure may be different; differences in the fast phase observed by temperature and pressure jump provide evidence for this. The infrared measurements that we performed in the amide I' region had phases similar to the time scales observed by fluorescence temperature and pressure jump, but with an additional $\sim 100$ ns phase. Fluorescence measurements are sensitive to the tryptophan side chain packing in the $\beta$-sheet of the first hairpin, whereas infrared measurements are sensitive to secondary structure changes in the peptide backbone. Because the $\sim 100 \mathrm{~ns}$ phase is located in the turn and the tryptophan is located in the $\beta$-sheet, the tryptophan may not report on dynamics in the turns. This phenomenon was also observed in temperature jump experiments probed by fluorescence and infrared spectroscopy in FBP28 WW domain. ${ }^{28}$ Our results support a hierarchical model of folding initiated in the turns, followed by formation of hairpin 1 and hairpin 2 . The fast phases are temperature independent and the slowest phase is highly temperature dependent, so as the temperature is raised the intermediate and slow phase overlap making it impossible to separate the two phases. Measurements of the aspartic acid side chain support a model with sequential intermediates; if the intermediates were formed along parallel nonsequential folding pathways, the aspartic acid would be protonated on more than one time scale and we would expect to observe the same multiexponential kinetics when $1710 \mathrm{~cm}^{-1}$ is probed as we observe for the other probed frequencies. The dynamics monitored at $1710 \mathrm{~cm}^{-1}$ are consistent with a hierarchal model where the side chain of aspartic acid is protonated in an intermediate along the folding pathway; however, this model does not eliminate the possibility of multiple folding pathways connecting these intermediate states. 
The FiP35 Pin1 WW domain mutant has a shortened first turn with aspartic acid based on the five-residue type-I G1 bulge turn found in FBP28 WW Domain. ${ }^{52}$ Infrared measurements of wildtype FBP28 WW domain show that the Asp in the turn is protonated, with a peak at $1715 \mathrm{~cm}^{-1}$ (Supporting Information Figure S7). This is consistent with a protonated carbonyl side chain, like we observe in FiP35 WW domain, engaged in hydrogen bonding with a neighboring side chain or protein backbone. We performed extensive kinetic measurements on a mutant of FBP28 WW Domain where the Asp was substituted for the neutral analogue Asn. ${ }^{28}$ The neutral analogue exhibited the same folding kinetics as the wildtype protein. This observation also supports our assignment of the $1713 \mathrm{~cm}^{-1}$ band in FiP35 to a hydrogen bonded carbonyl side chain of protonated aspartic acid, because Asn has an analogous carbonyl in the side chain that should be able to form the same hydrogenbonding network. The Gruebele and Kelly groups have demonstrated that shortening the first turn usually speeds folding and stabilizes the WW domain. ${ }^{26}$ While many of the variants they designed have an aspartic acid in the first turn, they also reported data for WT Pin1, which has no negatively charged residues in the turn. They observed a doubling of the folding rate and a 10 degree increase in $T_{\mathrm{m}}$ (indicating an increase in stability) by removing R12 of WT Pin1. Incorporation of the aspartic acid into the turn further stabilizes the mutants by 10 degrees and speeds folding by an additional $10 \mu \mathrm{s}$. In our T-jump measurements we observe that the aspartic acid is protonated in the intermediate step when the first loop is formed. The hydrogen bonds that aspartic acid forms stabilize the first turn, which lowers the barrier of the rate limiting formation of the second loop.

Extensive MD simulations of FiP35 generally agree that folding is more likely to proceed through formation of the first hairpin followed by the second hairpin; however, disagree on the presence of intermediates or downhill folding. ${ }^{29,31,59-61}$ The Pande group employed a distributed computing approach to run thousands of short simulations totaling $2.3 \mathrm{~ms}$, that showed heterogeneous folding of FiP35 with multiple folding pathways initiated in either sheet. ${ }^{62}$ Two $100 \mu$ s trajectories of FiP35 WW domain made available by D. E. Shaw Research predict downhill folding or folding through intermediates depending on the analysis method. ${ }^{31,60,61,63}$ The original work concluded that there is no barrier to folding. ${ }^{63}$ Krivov reanalyzed this data using a novel reaction coordinate and identified an intermediate where the first loop was formed. ${ }^{61}$ Markov-state-models (MSM) of the same data identified the presence of additional pathways, with a second pathway where the second loop is formed in the intermediate. ${ }^{31,60}$ Application of the kinetically discrimitory metric learning (KDML) algorithm to the MSM reveals two additional states on the submillisecond time scale. ${ }^{64}$ The faster state involves Asp13 flipping between a native hydrogen bond with Ser11 and nonnative hydrogen bonds with $\operatorname{Arg} 12$ and Arg16. While the aspartic acid is not protonated in these simulations, the identification of early dynamics at this position is consistent with our observation of a fast time scale intermediate involving protonation of the aspartic acid. A network based analysis supports these models, predicting that the pathway where the first loop is formed first is four times more probable. ${ }^{65}$ Monte Carlo simulations in two course-grained models support this model and further find that the probability of each pathway is dependent on starting temperature and initial condition. ${ }^{66}$ Three long time scale molecular dynamics simulations mimicking temperature jump conditions predict folding through the second hairpin; however, in one case the protein did not fully fold. ${ }^{29}$ Our results 
support models that predict folding through the first hairpin; however, none of the computational methods that have been applied to FiP35 have taken into account electrostatic interaction at Asp13. Optimization of the stability and folding kinetics of Pin1 WW domain was achieved with three charged residues located in the first turn. WW domains fold through an intermediate where the first hairpin forms first, so experimental and computational methods that capture electrostatic interactions are necessary to fully characterize the folding pathway. Much effort has been placed on developing computational methods that describe coupling between protein folding and $\mathrm{pH} .{ }^{67-70}$ Recently, all-atom continuous $\mathrm{pH}$ molecular dynamics simulations that determine the protonation state of titratable sites during molecular dynamics simulation at a specific $\mathrm{pH}$ have been under development by several groups. ${ }^{71-74}$ Applying these methods to FiP35 WW Domain it should be possible to not only predict the protein's $\mathrm{p} K_{\mathrm{a}}$ 's, but also determine the $\mathrm{pH}$-dependent conformational dynamics. ${ }^{75-79}$

\section{CONCLUSION}

Using infrared spectroscopy it is possible to monitor side chain dynamics with singleresidue specificity without incorporation of nonnative labels. We observe a protonated side chain in the first turn of the FiP35 WW domain with a $\mathrm{p} K_{\mathrm{a}}$ that is coupled to the folding. Time resolved measurements probed in the amide I region and at the aspartic acid side chain provide evidence for a hierarchical folding mechanism, similar to that proposed for other WW domains. ${ }^{17,25,27,28,30}$ Formation of the turns precedes formation of the first hairpin, with the final step being folding of the second hairpin. Infrared spectroscopy of the protonated side chain exhibits a single exponential that agrees with the intermediate phase, supporting the hierarchical folding model initiated in the first hairpin. Previous experimental and computational studies applied to this system lacked probes sensitive to the aspartic acid protonation state. These experiments demonstrate the importance of electrostatic interactions in the structural dynamics of even the smallest proteins.

\section{Supplementary Material}

Refer to Web version on PubMed Central for supplementary material.

\section{ACKNOWLEDGMENTS}

This work was supported by a grant from the National Institutes of Health (NIH R01 GM53640) to R.B.D.

\section{REFERENCES}

(1). Oliveberg M, Fersht AR. Biochemistry. 1996; 35:6795. [PubMed: 8639631]

(2). Stoycheva AD, Onuchic JN, Brooks CL. J. Chem. Phys. 2003; 119:5722.

(3). Waldburger CD, Jonsson T, Sauer RT. Proc. Natl. Acad. Sci. U. S. A. 1996; 93:2629. [PubMed: 8610092]

(4). Grimsley GR, Scholtz JM, Pace CN. Protein Sci. 2009; 18:247. [PubMed: 19177368]

(5). Kukic P, Farrell D, Sondergaard CR, Bjarnadottir U, Bradley J, Pollastri G, Nielsen JE. Proteins: Struct., Funct., Genet. 2010; 78:971. [PubMed: 19894279]

(6). Harris TK, Turner GJ. IUBMB Life. 2002; 53:85. [PubMed: 12049200]

(7). Engelhard M, Gerwert K, Hess B, Kreutz W, Siebert F. Biochemistry. 1985; 24:400. [PubMed: 3978081] 
(8). Barth A, Zscherp C. Q. Rev. Biophys. 2002; 35:369. [PubMed: 12621861]

(9). Bailey JA, Tomson FL, Mecklenburg SL, MacDonald GM, Katsonouri A, Puustinen A, Gennis RB, Woodruff WH, Dyer RB. Biochemistry. 2002; 41:2675. [PubMed: 11851414]

(10). McMahon BH, Fabian M, Tomson F, Causgrove TP, Bailey JA, Rein FN, Dyer RB, Palmer G, Gennis RB, Woodruff WH. Biochim. Biophys. Acta, Bioenerg. 2004; 1655:321.

(11). Taskent-Sezgin H, Chung JA, Banerjee PS, Nagarajan S, Dyer RB, Carrico I, Raleigh DP. Angew. Chem., Int. Ed. 2010; 49:7473.

(12). Macias MJ, Hyvonen M, Baraldi E, Schultz J, Sudol M, Saraste M, Oschkinat H. Nature. 1996; 382:646. [PubMed: 8757138]

(13). Macias MJ, Gervais V, Civera C, Oschkinat H. Nat. Struct. Biol. 2000; 7:375. [PubMed: 10802733]

(14). Verdecia MA, Bowman ME, Lu KP, Hunter T, Noel JP. Nat. Struct. Biol. 2000; 7:639. [PubMed: 10932246]

(15). Wiesner S, Stier G, Sattler M, Macias MJ. J. Mol. Biol. 2002; 324:807. [PubMed: 12460579]

(16). Piana S, Sarkar K, Lindorff-Larsen K, Guo MH, Gruebele M, Shaw DE. J. Mol. Biol. 2011; 405:43. [PubMed: 20974152]

(17). Jager M, Nguyen H, Crane JC, Kelly JW, Gruebele M. J. Mol. Biol. 2001; 311:373. [PubMed: 11478867]

(18). Ferguson N, Johnson CM, Macias M, Oschkinat H, Fersht A. Proc. Natl. Acad. Sci. U. S. A. 2001; 98:13002. [PubMed: 11687613]

(19). Karanicolas J, Brooks CL 3rd. Proc. Natl. Acad. Sci. U. S. A. 2003; 100:3954. [PubMed: 12655041]

(20). Karanicolas J, Brooks CL. Proc. Natl. Acad. Sci. U. S. A. 2004; 101:3432. [PubMed: 14981252]

(21). Mu YG, Nordenskiold L, Tam JP. Biophys. J. 2006; 90:3983. [PubMed: 16533840]

(22). Noe F, Schutte C, Vanden-Eijnden E, Reich L, Weikl TR. Proc. Natl. Acad. Sci. U. S. A. 2009; 106:19011. [PubMed: 19887634]

(23). Nguyen H, Jager M, Moretto A, Gruebele M, Kelly JW. Proc. Natl. Acad. Sci. U. S. A. 2003; 100:3948. [PubMed: 12651955]

(24). Ferguson N, Berriman J, Petrovich M, Sharpe TD, Finch JT, Fersht AR. Proc. Natl. Acad. Sci. U. S. A. 2003; 100:9814. [PubMed: 12897238]

(25). Petrovich M, Jonsson AL, Ferguson N, Daggett V, Fersht AR. J. Mol. Biol. 2006; 360:865. [PubMed: 16784750]

(26). Liu F, Du DG, Fuller AA, Davoren JE, Wipf P, Kelly JW, Gruebele M. Proc. Natl. Acad. Sci. U. S. A. 2008; 105:2369. [PubMed: 18268349]

(27). Davis CM, Dyer RB. J. Am. Chem. Soc. 2013; 135:19260. [PubMed: 24320936]

(28). Davis CM, Dyer RB. Biochemistry. 2014; 53:5476. [PubMed: 25121968]

(29). Wirth AJ, Liu Y, Prigozhin MB, Schulten K, Gruebele M. J. Am. Chem. Soc. 2015; 137:7152. [PubMed: 25988868]

(30). Deechongkit S, Nguyen H, Powers ET, Dawson PE, Gruebele M, Kelly JW. Nature. 2004; 430:101. [PubMed: 15229605]

(31). Lane TJ, Bowman GR, Beauchamp K, Voelz VA, Pande VS. J. Am. Chem. Soc. 2011; 133:18413. [PubMed: 21988563]

(32). Jaeger M, Nguyen H, Dendle M, Gruebele M, Kelly JW. Protein Sci. 2007; 16:1495. [PubMed: 17586778]

(33). Jager M, Dendle M, Kelly JW. Protein Sci. 2009; 18:1806. [PubMed: 19565466]

(34). Tremmel S, Beyermann M, Oschkinat H, Bienert M, Naumann D, Fabian H. Angew. Chem., Int. Ed. 2005; 44:4631.

(35). Andrushchenko VV, Vogel HJ, Prenner EJ. J. Pept. Sci. 2007; 13:37. [PubMed: 17031869]

(36). Williams S, Causgrove TP, Gilmanshin R, Fang KS, Callender RH, Woodruff WH, Dyer RB. Biochemistry. 1996; 35:691. [PubMed: 8547249]

(37). Manning MC, Woody RW. Biopolymers. 1987; 26:1731. [PubMed: 3663854]

$J$ Am Chem Soc. Author manuscript; available in PMC 2016 February 10. 
(38). Koepf EK, Petrassi HM, Ratnaswamy G, Huff ME, Sudol M, Kelly JW. Biochemistry. 1999; 38:14338. [PubMed: 10572009]

(39). Koepf EK, Petrassi HM, Sudol M, Kelly JW. Protein Sci. 1999; 8:841. [PubMed: 10211830]

(40). Viguera AR, Arrondo JLR, Musacchio A, Saraste M, Serrano L. Biochemistry. 1994; 33:10925. [PubMed: 8086409]

(41). Knapp S, Mattson PT, Christova P, Berndt KD, Karshikoff A, Vihinen M, Smith CIE, Ladenstein R. Proteins: Struct., Funct., Genet. 1998; 31:309. [PubMed: 9593201]

(42). Reid KL, Rodriguez HM, Hillier BJ, Gregoret LM. Protein Sci. 1998; 7:470. [PubMed: 9521124]

(43). Jager M, Dendle M, Fuller AA, Kelly JW. Protein Sci. 2007; 16:2306. [PubMed: 17766376]

(44). Nicholson EM, Scholtz JM. Biochemistry. 1996; 35:11369. [PubMed: 8784192]

(45). Yang WJ, Griffiths PR, Byler DM, Susi H. Appl. Spectrosc. 1985; 39:282.

(46). Arrondo JLR, Blanco FJ, Serrano L, Goni FM. FEBS Lett. 1996; 384:35. [PubMed: 8797798]

(47). Susi H, Byler DM. Methods Enzymol. 1986; 130:290. [PubMed: 3773736]

(48). Wang T, Xu Y, Du DG, Gai F. Biopolymers. 2004; 75:163. [PubMed: 15356870]

(49). Hilario J, Kubelka J, Keiderling TA. J. Am. Chem. Soc. 2003; 125:7562. [PubMed: 12812496]

(50). Maness SJ, Franzen S, Gibbs AC, Causgrove TP, Dyer RB. Biophys. J. 2003; 84:3874. [PubMed: 12770893]

(51). Ranganathan R, Lu KP, Hunter T, Noel JP. Cell. 1997; 89:875. [PubMed: 9200606]

(52). Jager M, Zhang Y, Bieschke J, Nguyen H, Dendle M, Bowman ME, Noel JP, Gruebele M, Kelly JW. Proc. Natl. Acad. Sci. U. S. A. 2006; 103:10648. [PubMed: 16807295]

(53). Kubelka J, Keiderling TA. J. Am. Chem. Soc. 2001; 123:12048. [PubMed: 11724613]

(54). Deacon GB, Phillips RJ. Coord. Chem. Rev. 1980; 33:227.

(55). Urry DW, Peng SQ, Parker TM, Gowda DC, Harris RD. Angew. Chem., Int. Ed. Engl. 1993; 32:1440.

(56). Langsetmo K, Fuchs JA, Woodward C. Biochemistry. 1991; 30:7603. [PubMed: 1854757]

(57). Langsetmo K, Fuchs JA, Woodward C, Sharp KA. Biochemistry. 1991; 30:7609. [PubMed: 1906744]

(58). Auzat I, Garel JR. Protein Sci. 1992; 1:254. [PubMed: 1304907]

(59). Lindorff-Larsen K, Piana S, Dror RO, Shaw DE. Science. 2011; 334:517. [PubMed: 22034434]

(60). Kellogg EH, Lange OF, Baker DJ. Phys. Chem. B. 2012; 116:11405.

(61). Krivov SV. J. Phys. Chem. B. 2011; 115:12315. [PubMed: 21902225]

(62). Ensign DL, Pande VS. Biophys. J. 2009; 96:L53. [PubMed: 19383445]

(63). Shaw DE, Maragakis P, Lindorff-Larsen K, Piana S, Dror RO, Eastwood MP, Bank JA, Jumper JM, Salmon JK, Shan Y, Wriggers W. Science. 2010; 330:341. [PubMed: 20947758]

(64). McGibbon RT, Pande VS. J. Chem. Theory Comput. 2013; 9:2900. [PubMed: 26583974]

(65). Berezovska G, Prada-Gracia D, Rao F. J. Chem. Phys. 2013; 139:035102. [PubMed: 23883056]

(66). S AB, Skrbic T, Covino R, Faccioli P. Proc. Natl. Acad. Sci. U. S. A. 2012; 109:2330. [PubMed: 22308345]

(67). Ripoll DR, Vorobjev YN, Liwo A, Vila JA, Scheraga HA. J. Mol. Biol. 1996; 264:770. [PubMed: 8980685]

(68). Yang AS, Honig B. J. Mol. Biol. 1993; 231:459. [PubMed: 8510157]

(69). Gilson MK. Proteins: Struct., Funct., Genet. 1993; 15:266. [PubMed: 8456096]

(70). You TJ, Bashford D. Biophys. J. 1995; 69:1721. [PubMed: 8580316]

(71). Donnini S, Tegeler F, Groenhof G, Grubmuller H. J. Chem. Theory Comput. 2011; 7:1962. [PubMed: 21687785]

(72). Goh GB, Knight JL, Brooks CL 3rd. J. Chem. Theory Comput. 2012; 8:36. [PubMed: 22337595]

(73). Wallace JA, Shen JK. J. Chem. Phys. 2012; 137:184105. [PubMed: 23163362]

(74). Lee J, Miller BT, Damjanovic A, Brooks BR. J. Chem. Theory Comput. 2014; 10:2738. [PubMed: 25061443]

(75). Wallace JA, Wang Y, Shi C, Pastoor KJ, Nguyen BL, Xia K, Shen JK. Proteins: Struct., Funct., Genet. 2011; 79:3364. [PubMed: 21748801] 
(76). Arthur EJ, Yesselman JD, Brooks CL 3rd. Proteins: Struct., Funct., Genet. 2011; 79:3276. [PubMed: 22002886]

(77). Liu H, Huo S. J. Phys. Chem. B. 2012; 116:646. [PubMed: 22126397]

(78). Laricheva EN, Arora K, Knight JL, Brooks CL 3rd. J. Am. Chem. Soc. 2013; 135:10906. [PubMed: 23841875]

(79). Maupin CM, Wong KF, Soudackov AV, Kim S, Voth GA. J. Phys. Chem. A. 2006; 110:631. [PubMed: 16405335] 


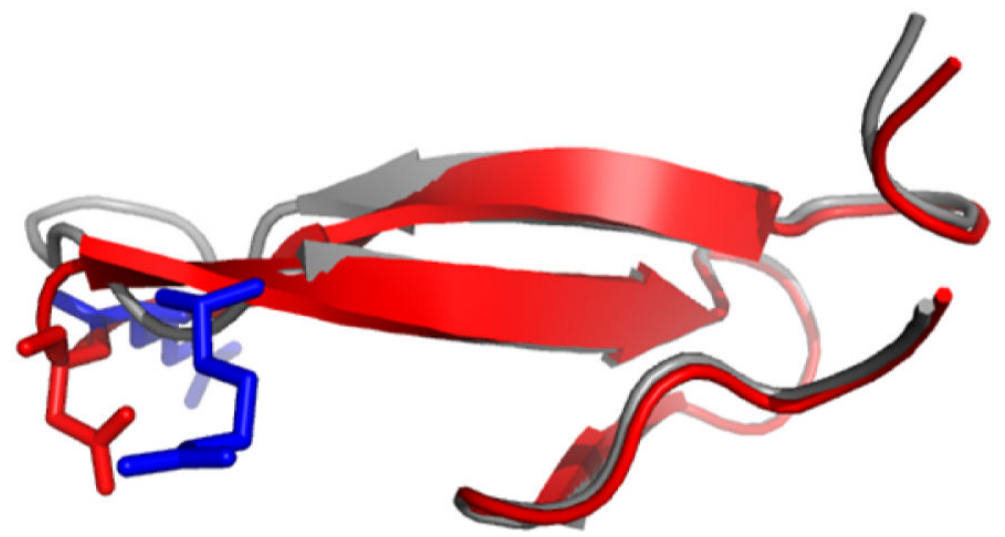

Figure 1.

Aligned three-dimensional models of Pin1 (gray) and FiP35 (red) WW Domains obtained by $\mathrm{X}$-ray diffraction. Arginines (blue) and aspartic acid (red) in the FiP35 sequence are displayed as sticks. (PDB entries: 1PIN, 2F21). The figure was prepared using PyMOL (www.pymol.org). 

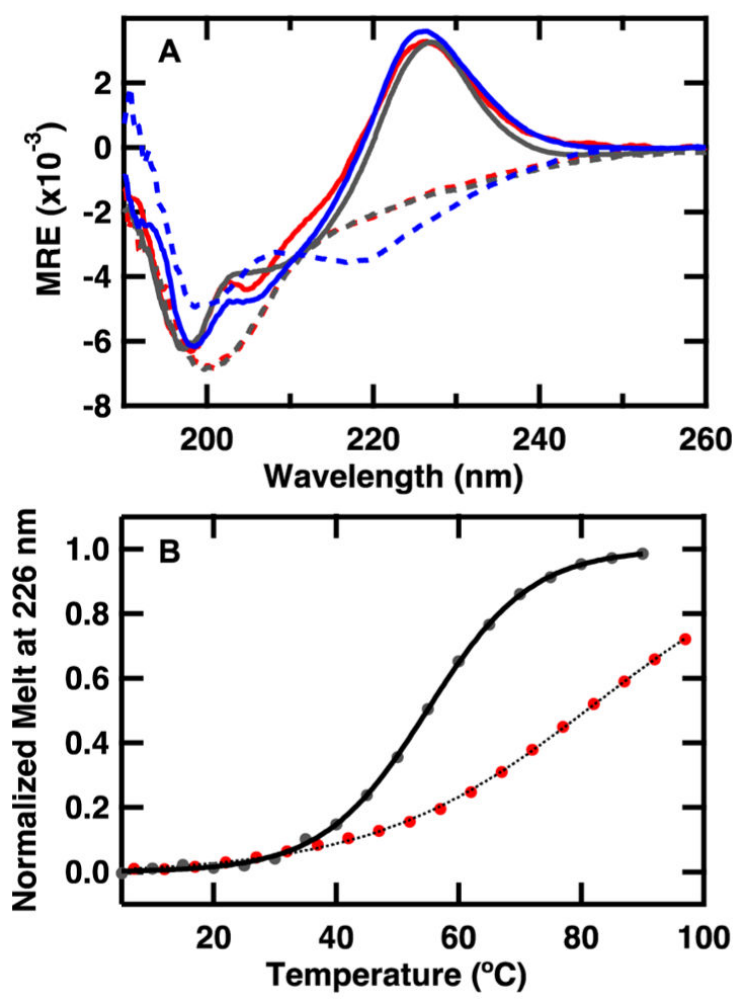

Figure 2.

(A) Far-UV CD spectra of $50 \mu \mathrm{M}$ solutions of the FiP35 (red), $\Delta \Delta$ FiP35 (blue) and Pin1 (gray) WW domains in $20 \mathrm{mM}$ potassium phosphate buffer $\left(\mathrm{pH} \mathrm{7.0)}\right.$ ) acquired at $5{ }^{\circ} \mathrm{C}(-)$ and $95{ }^{\circ} \mathrm{C}$ (---) during the course of a thermal denaturation in a $0.1 \mathrm{~cm}$ path length cell. (B) Thermal denaturation of the WW domains monitored by CD at $226 \mathrm{~nm}$. The continuous line represents the best fit to the data to a sigmoid (eq 2). 


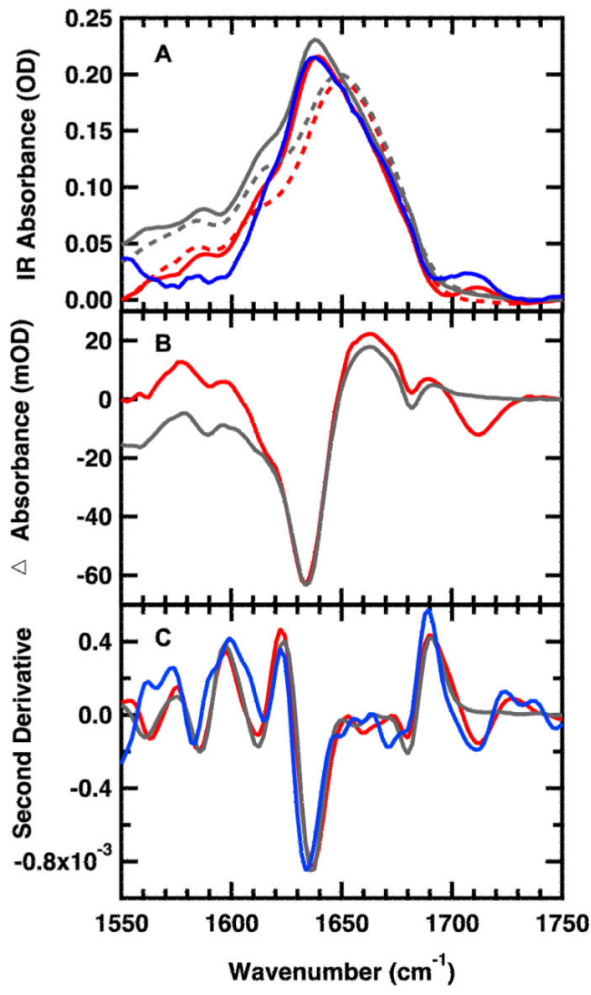

Figure 3.

Temperature dependent FTIR spectra of $1.5 \mathrm{mM}$ FiP35 (red), $50 \mu \mathrm{M} \Delta \Delta \mathrm{FiP} 35$ (blue) and 1.5 mM Pin1 (gray) WW Domains in $20 \mathrm{mM}$ potassium phosphate buffer (pH 7). (A)

Absorption spectra in the Amide I' region, acquired at $20^{\circ} \mathrm{C} \mathrm{(-)} \mathrm{and} 75{ }^{\circ} \mathrm{C}(---)$ during the course of a thermal denaturation. Spectra are normalized at $1636 \mathrm{~cm}^{-1}$ for comparison. (B) Difference spectra obtained by subtracting the spectrum at $20^{\circ} \mathrm{C}$ from the spectra at $75{ }^{\circ} \mathrm{C}$ and normalized at $1636 \mathrm{~cm}^{-1}$ for comparison. (C) Normalized second derivative of FTIR spectra at $5{ }^{\circ} \mathrm{C}$. 

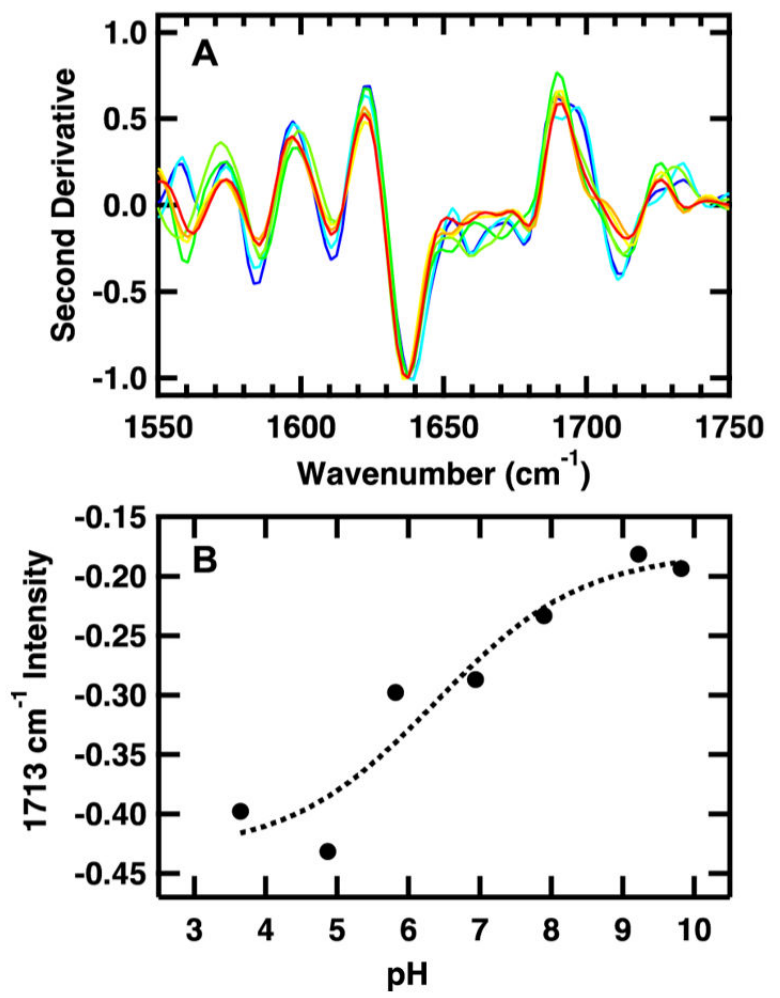

Figure 4.

pH dependent FTIR spectra of $0.8 \mathrm{mM}$ FiP35 WW Domain in buffer (see Experimental Section for buffer $\mathrm{pH} 4-10$ ) at $20^{\circ} \mathrm{C}$. (A) Normalized second derivative of FTIR spectra $\mathrm{pH}$ 4-10 (blue-red). (B) pH titration of protonated carboxylic acid obtained by plotting the change in IR second derivative spectra at $1713 \mathrm{~cm}^{-1}$ versus $\mathrm{pH}$. The data are fit to an apparent 2-state model (eq 2). 


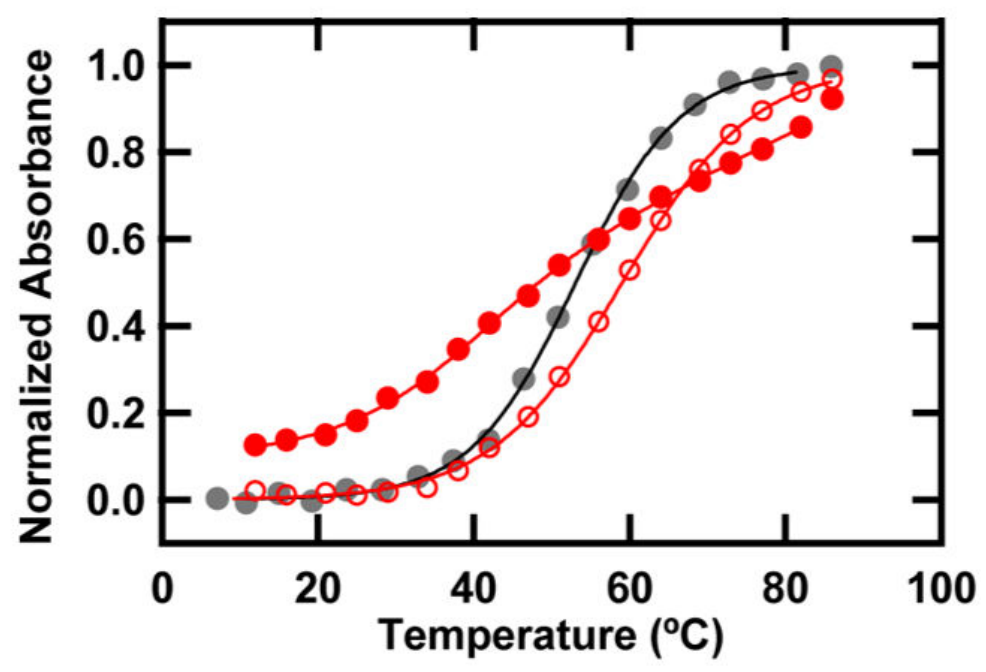

Figure 5.

FTIR melt curves for the FiP35 (red) and Pin1 (gray) WW domains obtained by plotting the change in IR difference spectra at $1636 \mathrm{~cm}^{-1}$ (closed circles) and $1713 \mathrm{~cm}^{-1}$ (open circles) versus temperature. The data are fit to an apparent 2 -state model and then normalized (eq 2). 


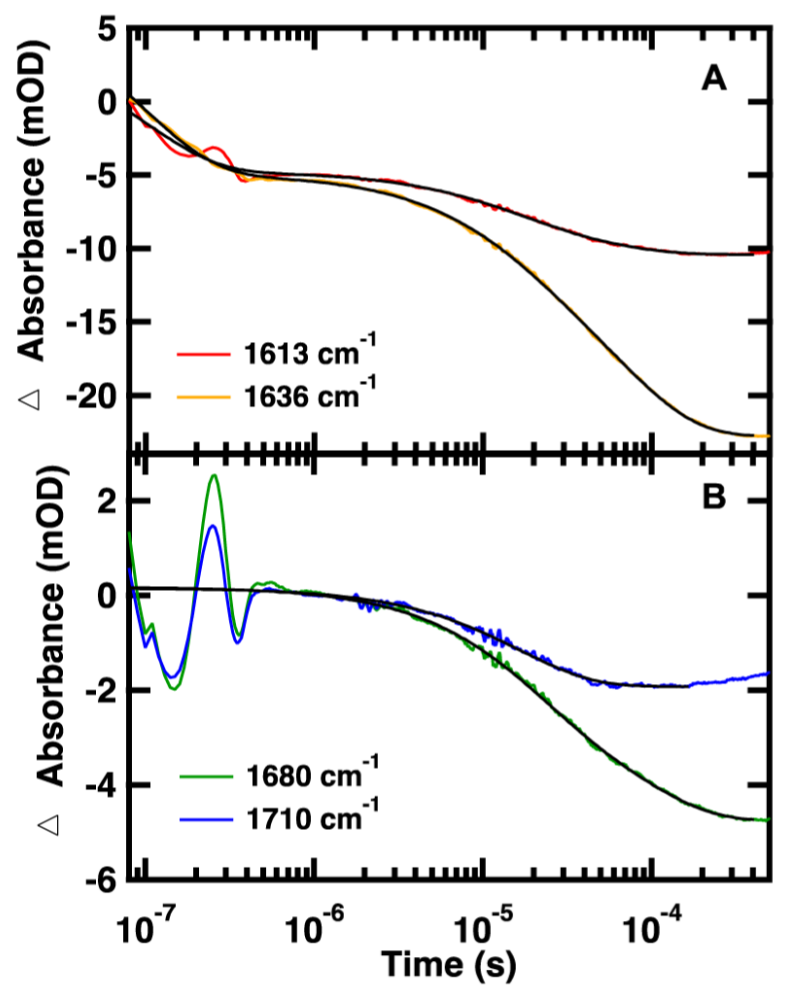

Figure 6.

Representative IR T-jump relaxation kinetics of $2.0 \mathrm{mM}$ FiP35 in $20 \mathrm{mM}$ potassium phosphate buffer ( $\mathrm{pH} 7$ ) monitored in the amide I' spectral region at 1613, 1636, and 1680 $\mathrm{cm}^{-1}$ and the protonated carboxylic acid side chain of aspartic acid at $1710 \mathrm{~cm}^{-1}$ following a T-jump from 35 to $50{ }^{\circ} \mathrm{C}$. A triple exponential fit is overlaid on the 1613 and $1636 \mathrm{~cm}^{-1}$ kinetic trace, double exponential fit is overlaid on the $1680 \mathrm{~cm}^{-1}$ kinetic trace and a single exponential fit is overlaid on the $1710 \mathrm{~cm}^{-1}$ kinetic trace (eq 1). 


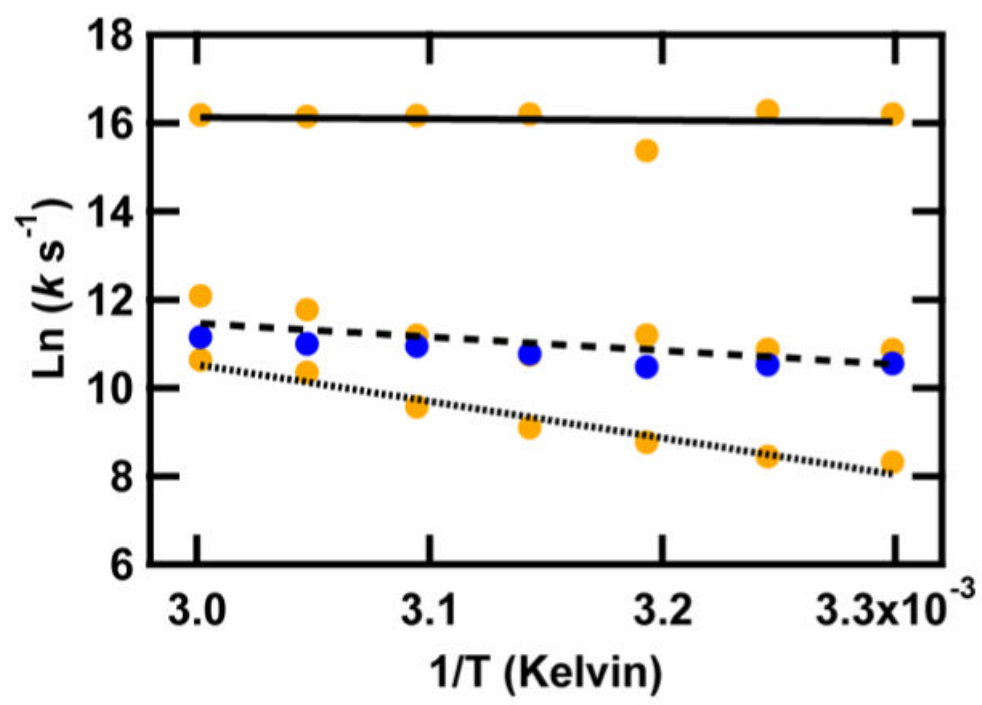

Figure 7.

Arrhenius plot showing the temperature dependence of the folding region at $1636 \mathrm{~cm}^{-1}$ (orange) and $1710 \mathrm{~cm}^{-1}$ (blue). The values of $T$ used for the $(1 / T)$ axis are the final temperatures reached during the jump. $k$ is the value obtained from a fit of the T-jump transient of FiP35. Lines are a result of fitting $\tau_{1}$ (solid line), $\tau_{2}$ (dashed line), and $\tau_{3}$ (dotted line) of FiP35. 


\section{Table 1}

WW Domain Sequences and Stability ${ }^{a}$

\begin{tabular}{|l|l|l|}
\hline WW Domain & Sequence & Melting Temperature $\left({ }^{\circ} \mathbf{C}\right)$ \\
\hline WTPinl & KLPPGWEKRMSRSSGRVYYFNHITNASQWERPSG & $59^{32}$ \\
\hline FiP35 Pinl & KLPPGWEKRMSR-DGRVYYFNHITNASQFERPSG & $78^{26}$ \\
\hline R12A R16A $\triangle \Delta$ FiP35 & KLPPGWEKRMSA-DGAVYYFNHITNASQFERPSG & -- \\
\hline
\end{tabular}

$a_{\beta \text {-sheets are underlined, mutations are bold, turn residues with positive (blue) and negative charges (red). }}$ 
Table 2

Relaxation Kinetics Following a Jump from 35 to $50{ }^{\circ} \mathrm{C}$

FiP35 WW domain

\begin{tabular}{lcccclc}
\hline & $\mathbf{A}_{\mathbf{1}}(\mathbf{m O D})$ & $\tau_{\mathbf{1}}(\mathbf{n s})$ & $\mathbf{A}_{\mathbf{2}}(\mathbf{m O D})$ & $\tau_{\mathbf{2}}(\boldsymbol{\mu} \mathbf{s})$ & $\mathbf{A}_{\mathbf{3}}(\mathbf{m O D})$ & $\tau_{\mathbf{3}}(\boldsymbol{\mu} \mathbf{s})$ \\
$1613 \mathrm{~cm}^{-1}$ & $3.4 \pm 0.1$ & $108 \pm 4$ & $3.6 \pm 0.2$ & $14.7 \pm 0.7$ & $2.1 \pm 0.2$ & $56 \pm 6$ \\
$1636 \mathrm{~cm}^{-1}$ & $5.0 \pm 0.1$ & $95 \pm 2$ & $4.8 \pm 0.1$ & $13.9 \pm 0.2$ & $13.0 \pm 0.1$ & $70 \pm 1$ \\
$1680 \mathrm{~cm}^{-1}$ & & & $2.5 \pm 0.2$ & $18 \pm 1$ & $2.5 \pm 0.2$ & $86 \pm 8$ \\
$1710 \mathrm{~cm}^{-1}$ & & & $1.9 \pm 0.1$ & $17 \pm 1$ & & \\
\hline
\end{tabular}

